\title{
The status of the cervical spine in preschool children with a history of congenital muscular torticollis
}

\author{
Anna M. Öhman \\ Department of Paediatrics, University of Gothenburg, Queen Silvia Children's Hospital, Gothenburg, Sweden; \\ anna.ohman@,friskispraktiken.com
}

Received 6 September 2013; revised 8 October 2013; accepted 15 October 2013

Copyright (C) 2013 Anna M. Öhman. This is an open access article distributed under the Creative Commons Attribution License, which permits unrestricted use, distribution, and reproduction in any medium, provided the original work is properly cited.

\section{ABSTRACT}

Background: Infants with congenital muscular torticollis are born with an asymmetric range of motion and a muscular imbalance in the cervical spine, as a result of a shortening or excessive contraction of the sternocleidomastoid muscle. Purpose: The study aimed to investigate passive range of motion (PROM) for rotation and lateral flexion, and muscle function of the cervical spine in children that had a history of CMT as infants. Study design: a prospective cohort study. Patient sample: 58 children at the age of 3.5 to 5 years that had been treated for CMT have infants participated in the study. Method: PROM was measured with protractors and muscle function was estimated with a modified Muscle Function Scale. Data from infancy were taken from earlier records. Result: PROM in rotation of the neck was mean $98.7^{\circ}$ and PROM in lateral flexion of the neck was mean $69.1^{\circ}$. Symmetric PROM of the neck was found in $74 \%$ of the children for rotation and in $88 \%$ of the children for lateral flexion. Multiple regression showed that gender and PROM in rotation as infants had a significant impact on asymmetric PROM. Forty-five percent of the children had some degree of muscular imbalance in the lateral flexors of the neck. Conclusion: Possible risk factors for later asymmetric PROM are: gender, birth weight, gestation week and PROM in rotation as infants. These factors ought to be taken into consideration when developing guidelines for long-term follow-up.

Keywords: Congenital Muscular Torticollis; Cervical Spine; Passive Range of Motion; Rotation; Muscle Function; Children

\section{INTRODUCTION}

Congenital muscular torticollis (CMT) is a result of shortening or excessive contraction of the sternocleidomastoid muscle, often with limited Range of Motion (ROM) of the neck, in rotation on the affected side and in lateral flexion on the non-affected side. For infants/ children with CMT there is also an imbalance in muscle function i.e. they have a lack of muscular strength and endurance around the neck on the non-affected side and sometimes an excessive muscular strength on the affected side $[1,2]$. This imbalance is not found in healthy infants [3]. The reported incidence of CMT is $0.4 \%$ $2.0 \%$ [1], however the incidence may be higher as Stellwagen et al. found that $16 \%$ of newborns had torticollis [4]. The cause of CMT has been discussed but it seems likely to be the sequela of intrauterine or perinatal compartment syndrome $[5,6]$. Treatment mostly gives an excellent or good result within the first year. However, the child is a growing individual and this fact may change the status of the cervical spine over the years. An excellent treatment result when the child is less than one year old may not last long-term. At later ages some children are found to be in need of surgery in spite of an excellent or good result as an infant. We need more knowledge about the status of the cervical spine for the growing child with a history of CMT.

The reference values for Passive ROM (PROM) in rotation of the neck for healthy children aged 3.5 to 5 years have been shown to be mean $100.1^{\circ}\left(\mathrm{SD} 7.7^{\circ}\right)$ [7]. This is ten degrees less than for healthy infants with a mean of $110^{\circ}\left(\mathrm{SD} 6.2^{\circ}\right.$ ) [3]. There seems to be a natural loss of about $10^{\circ}$ rotation of the neck up to preschool age. PROM in lateral flexion of the neck for healthy children aged 3.5 to 5 year is mean $68.5^{\circ}$ (SD 3.4); this is very close to the mean for healthy infants which is $70^{\circ}$ (SD 2.2). This indicates that PROM for rotation decreases and PROM in lateral flexion stays almost the same during the first five years of life [7]. The aim of this study 
was to investigate PROM in rotation and lateral flexion of the neck and to investigate whether PROM is symmetric or asymmetric in children aged 3.5 to 5 years who had a history of CMT. Also to see if PROM at the start of treatment for CMT, age at the start of treatment, gender and current head tilt, muscular imbalance, remaining plagiocephaly, birth weight and gestation week had any influence on the PROM at the age of 3.5 to 5 years. And also to investigate if there were any detectable differences in muscle function/strength between the right and left sides in the lateral flexor muscles of the neck. The local ethical committee approved the study and the parents gave their informed consent.

\section{METHOD}

Neck rotation was measured with an arthrodial protractor [1]. The child was lying supine on the examination table with the shoulders stabilized. The examiner supported the head and neck in the neutral position, over the edge of the examination table. In this position the neck could be rotated and moved freely in all directions. According to Cheng et al. there is an inter-examiner reliability correlation coefficient of 0.71 for neck ROM in infants [1].

Lateral flexion was measured with the child lying in supine on a large protractor with the shoulders stabilized $[2,8]$. This method was found to have a high intra-rater reliability of ICC $0.94-0.98$ [9]. The maximal values for PROM in rotation and lateral flexion of the cervical spine were recorded; both left and right sides were measured.

Muscle function/strength of the lateral flexor muscles of the neck was estimated by using the same technique as when tested with the Muscle Function Scale [10]. Holding the child horizontally around the trunk without support for the head, the child was asked to lift the head as high as possible. A modified method to score was used: no difference (score 0), marginal (score 1), distinct (score 2) or extensive difference (score 3 ) between right and left sides. Head tilt and plagiocephaly i.e. posterior flattening of the skull were also recorded similarly with scores 0 to 3. Data from infancy were taken from earlier records. The same paediatric physiotherapist was responsible for carrying out all the measurements on all children.

\section{STATISTICS}

The mean, the standard deviation (SD) and the ranges were calculated for PROM in rotation and lateral flexion. Differences between children with asymmetric and symmetric PROM at their current age were assessed by analysis of covariance (ANCOVA), using the degree of asymmetric PROM as an infant, age at the start of treat- ment as an infant and current MFS scores, head tilt, plagiocephaly, age and gender as covariates. Any differences in mean weight at birth and mean gestation week between children with asymmetric and symmetric PROM were investigated with the Mann Whitney test. The SPSS statistical programme was used and p-values of 0.05 or less were considered evidence of statistically significant findings.

\section{RESULT}

Fifty-eight children participated 25 female and 33 male, they were at a mean age of 4.3 years SD 0.57 (range 3.5 - 5 years). PROM in rotation was measured for 57 children and lateral flexion for 58 children. One child was not investigated for rotation, as he did not want to cooperate in this measurement. PROM in rotation of the neck was mean $98.7^{\circ}$, SD $8.4^{\circ}$ and PROM in lateral flexion of the neck was mean $69.1^{\circ}$, SD $4.2^{\circ}$. Symmetric PROM of the neck was found in $74 \%$ of the children for rotation and in $88 \%$ of the children for lateral flexion. Twenty-six percent of the children had asymmetric PROM in rotation and/or lateral flexion of the neck (Table 1); six children had asymmetry in both motions, eight children only in rotation and one child only in lateral flexion. Multiple regressions showed that both PROM in rotation as infants and gender (female) had a significant impact on asymmetric PROM at the age of 3.5 - 5 years (Tables 2 and 3). Twenty-six (45\%) of the children had some degree of muscular imbalance in the lateral flexors of the neck. Fourteen (54\%) had marginal imbalance, eight $(31 \%)$ had distinct imbalance and four (15\%) had extensive imbalance in the lateral flexors of the neck.

Three children had some degree of head tilt, two minor and one moderate, the latter was in need of surgery because of a muscular string and limited PROM in both rotation and lateral flexion. She had an excellent result after surgery.

Four children had minor plagiocephaly. There was a trend that children with asymmetric PROM at the age of 3.5 - 5 years were born slightly later and had higher birth weight than children with symmetric PROM (Table 4). These differences were not significant.

\section{DISCUSSION}

In the current study the majority of the children who had CMT as infants had excellent PROM for rotation and lateral flexion of the neck at the age of 3.5 to 5 years. The mean PROM for both rotation and lateral flexion of the neck in this study were very close to the reference values for healthy children who had not had neck problems as infants [7]. Twenty-six percent of the children had some degree of asymmetry in PROM, however as the children were rather young at the time of the assess- 
Table 1. Data for the seven females and eight males who had asymmetric PROM, only four children had less than $90^{\circ}$ in rotation of the neck.

\begin{tabular}{|c|c|c|c|c|c|c|c|c|}
\hline Child & $\begin{array}{l}\text { Rotation } \\
\text { left-right }\end{array}$ & $\begin{array}{c}\text { Rotation difference } \\
\text { between sides in degrees }\end{array}$ & $\begin{array}{l}\text { Lateral flexion difference } \\
\text { between sides in degrees }\end{array}$ & Gestation week & $\begin{array}{l}\text { MFS difference in } \\
\text { scores between sides }\end{array}$ & Tilt degree & $\begin{array}{c}\text { Age at } \\
\text { assessment }\end{array}$ & Gender \\
\hline I & $100-110$ & 10 & 0 & 43 & 0 & 0 & 4.5 & M \\
\hline II & $100-90$ & 10 & 5 & 43 & 1 & 0 & 5 & M \\
\hline III & $110-100$ & 10 & 10 & 42.6 & 1 & 2 & 4.5 & M \\
\hline IV & $105-100$ & 5 & 5 & 42.8 & 0 & 0 & 4 & M \\
\hline V & $90-85$ & 5 & 10 & 38.1 & 3 & 0 & 4 & M \\
\hline VI & $95-95$ & 0 & 5 & 40.7 & 1 & 0 & 5 & M \\
\hline VII & $85-100$ & 15 & 5 & 42 & 1 & 0 & 4.5 & $\mathrm{~F}$ \\
\hline VIII & $110-120$ & 10 & 0 & 39 & 2 & 0 & 5 & F \\
\hline IX & $85-100$ & 15 & 10 & 40 & 3 & 2 & 4 & $\mathrm{~F}$ \\
\hline $\mathrm{X}$ & $85-90$ & 5 & 0 & 41.4 & 1 & 3 & 3.5 & M \\
\hline XI & $95-100$ & 5 & 0 & 41 & 1 & 1 & 3.5 & $\mathrm{~F}$ \\
\hline XII & $90-95$ & 5 & 0 & 40.3 & 1 & 0 & 3.5 & M \\
\hline XIII & $90-95$ & 5 & 0 & 39.6 & 0 & 0 & 5 & $\mathrm{~F}$ \\
\hline XIV & $100-110$ & 10 & 0 & 38 & 0 & 0 & 4 & $\mathrm{~F}$ \\
\hline XV & $90-100$ & 10 & 0 & 39.7 & 2 & 0 & 5 & $\mathrm{~F}$ \\
\hline
\end{tabular}

Table 2. Multiple regression, effects of covariates, the PROM as infants and gender had a significant impact on PROM at the age of 3.5 - 5 years.

\begin{tabular}{ccccc}
\hline & Standard error & Beta & $95 \%$ confidence interval & p-value \\
\hline PROM as infant & 0.035 & 0.579 & $0.079-0.223$ & $<0.001$ \\
Current MFS scores & 0.882 & 0.129 & $-0.954-2.621$ & 0.351 \\
Head tilt at age 3.5 - 5 years & 1.430 & 0.167 & $-1.055-4.745$ & 0.205 \\
Remaining plagiocephaly at age 3.5 - 5 years & 3.067 & 0.037 & $-5.432-7.010$ & 0.799 \\
Current age & 0.981 & -0.019 & $-2.136-1.842$ & 0.881 \\
Treatment start as infant & 0.450 & 0.056 & $-0.741-1.083$ & 0.706 \\
Gender & 1.219 & -0.291 & $-5.073--0.130$ & 0.040 \\
\hline
\end{tabular}

Table 3. This shows that females had higher frequency of asymmetric PROM in rotation than males in the current study.

\begin{tabular}{ccc}
\hline & Female & Male \\
\hline Asymmetric PROM for rotation in any degree & $28 \%$ & $25 \%$ \\
Asymmetric PROM for rotation with at least 10 & $20 \%$ & $9 \%$ \\
Asymmetric PROM for rotation with $15^{\circ}$ & $8 \%$ & $0 \%$ \\
\hline
\end{tabular}

ment, the risk of long-term effects cannot be excluded yet. As PROM in rotation at the start of the treatment as infants was found to have a significant impact on asymmetric PROM at the age of 3.5 - 5 years, this ought to be
Table 4. There was a trend towards higher birth weight and later gestation week in children with asymmetric PROM at the age of 3.5 - 5 years.

\begin{tabular}{ccc}
\hline At birth & $\begin{array}{c}\text { Children with } \\
\text { asymmetric PROM at } \\
\text { age 3.5 - 5 years }\end{array}$ & $\begin{array}{c}\text { Children with } \\
\text { symmetric PROM at } \\
\text { age 3.5 - 5 years }\end{array}$ \\
\hline Gestation week mean & 40.7 & 40.0 \\
Birth weight mean & 3694 grams & 3478 grams \\
\hline
\end{tabular}

taken into consideration when developing guidelines for long-term follow-up. Of the 15 children with asymmetric PROM for rotation only four had less than $90^{\circ}$ in the 
limited direction, this is more than adult PROM for rotation. For children in need of surgery there is often also a muscular band that limits the motion. There may not be a problem if a child has asymmetric but large PROM and no muscular band, but it may still be important to observe the child for possible long-term effects. Chang et al. found that ROM in lateral flexion is limited in those cases that need surgery. Unfortunately, in the studies that were found about long-term follow-up [2,5,11,12], there is a lack of information about raw data and how the measurements were performed. This makes it difficult to compare with the current study. Later gestation week and higher birth weight may be risk factors for later problems with asymmetric PROM for children with CMT. Chen et al. screened 1021 newborn infants for CMT with sonography and found that the infants with CMT were statistically significantly longer and heavier than the normative group [13]. It is common that mothers of infants with CMT state that the infant was in the birth canal for a rather long time before birth. Intrauterine malposition is one possible cause for CMT; both old and recent studies support this theory $[4,5,14,15]$.

In the current study $21 \%$ still had obvious muscular imbalance in the lateral flexors of the neck. For those children with head tilt it was more common with muscular imbalance than with asymmetric PROM. There is a need for strategies of how to best prevent later problems due to muscular imbalance. At the time of the study six children had minor tilt that was not a problem. However, the parents have to be observant to notice if it becomes worse as the child gets older. For the child who had surgery after the study, the parents were not aware of the problem before the study, but could after the assessment not understand how they could have missed the obvious head tilt and muscular band. This reflects that the parents can become blind to flaws and miss a very obvious head tilt. We usually tell the parents to observe the child's head position once or twice a year until the child stops growing; they also receive this information in writing. We have to consider how we can further improve the information. Chang et al. suggest that infants with CMT ought to be followed up until four years of age [5].

\section{LIMITATIONS}

MFS are developed for infants and are not adjusted for children of an older age. To use it in a modified version demands experience when assessing children at this age for this diagnosis. An alternative would be a handheld dynamometer, but clinical experience has shown that it is less reliable in younger ages. However for older children, from about 6 years, the dynamometer seems to function well $[2,8]$.

\section{CONCLUSION}

The PROM for rotation as infants, gender and also birth weight and gestation week may be risk factors for later asymmetric PROM in lateral flexion and rotation of the neck. Muscular imbalance in the lateral flexors of the neck but not necessary asymmetric PROM seems to be a risk factor for a later tendency of head tilt. This ought to be taken into consideration when developing guidelines for long-term follow-up.

\section{REFERENCES}

[1] Cheng, J.C.Y., Wong, M.W.N., Tang, S.P., Chen, T.M.K., Shum, S.L.F. and Wong, M.A. (2001) Clinical determinants of the outcome of manual stretching in the treatment of congenital muscular torticollis in infants. Journal of Bone and Joint Surgery, 83, 679-687.

[2] Öhman, A. and Beckung, E. (2005) Functional and cosmetic status in children treated for congenital muscular torticollis as infants. Advances in Physiotherapy, 7, 135140. http://dx.doi.org/10.1080/14038190500213836

[3] Öhman, A. and Beckung, E. (2008) Reference values for range of motion and muscle function in the neck-In infants. Pediatric Physical Therapy, 20, 53-58. http://dx.doi.org/10.1097/PEP.0b013e31815ebb27

[4] Stellwagen, L., Hubbard, E., Chambers, C. and Lyons, J.K. (2008) Torticollis, facial asymmetry and plagiocephaly in normal newborns. Archives of Disease in Childhood, 10, 827-831. http://dx.doi.org/10.1136/adc.2007.124123

[5] Lee, Y.T., Cho, S.K., Yoon, K., et al. (2011) Risk factors for intrauterine constraint are associated with ultrasonographically detected severe fibrosis in early congenital muscular torticollis. Journal of Pediatric Surgery, 46, 514-519.

http://dx.doi.org/10.1016/j.jpedsurg.2010.08.003

[6] Chang, P.Y., Tan, C.K., Huang, Y.F., Sheu, J.C., Wang, N.L., Yeh, M.L. and Chen, C.C. (1996) Torticollis: A long-term follow-up study. Zhonghua Min Guo Xiao Er Ke Yi Xue Hui Za Zhi, 37, 173-177.

[7] Öhman, A. and Beckung, E. (2012) A pilot study on changes in passive range of motion in the cervical spine, for children aged 0 - 5 years. Physiotherapy Theory and Practice, 29, 457-460.

http://dx.doi.org/10.3109/09593985.2012.753495

[8] Öhman, A., Perbeck, K.E., Beckung, E. and HaglundÅkerlind, Y. (2006) Functional and cosmetic status after surgery in congenital muscular torticollis. Advances in Physiotherapy, 8, 182-187. http://dx.doi.org/10.1080/14038190600780213

[9] Perbeck, K.E., Elfving, B., Haglund, Y. and Brogren, C.E. (2005) Intra-rater reliability in measuring range of motion in infants with congenital muscular torticollis. Advances in Physiotherapy, 7, 84-91. http://dx.doi.org/10.1080/14038190510010331

[10] Öhman, A., Nilsson, S. and Beckung, E. (2009) Validity and reliability of the Muscle Function Scale, aimed to assess the lateral flexors of the neck in infants. Physiotherapy Theory and Practice, 25, 129-137. http://dx.doi.org/10.1080/09593980802686904

[11] Binder, H., Eng, G.D., Gaiser, J.F. and Koch, B. (1987) 
Congenital muscular torticollis: Result of conservative management with long-term follow-up in 85 cases. Archives of Physical Medicine and Rehabilitation, 68, 222-225.

[12] Canale, S.T., Griffin, D.W. and Hubbard, C.N. (1982) Congenital muscular torticollis. Journal of Bone and Joint Surgery, 64, 810-816.

[13] Chen, M.M., Chang, H.C., Hisieh, C.F., Yen, M.F. and Chen, T.H.H. (2005) Predictive model for congenital muscular torticollis: Analysis of 1021 infants with sono- graphy. Archives of Physical Medicine and Rehabilitation, 86, 2199-2203.

http://dx.doi.org/10.1016/j.apmr.2005.05.010

[14] Jones, P. (1968) Torticollis in infancy and childhood. Charles C Thomas, Springfield, 17-21.

[15] Davis, J., Wenger, D. and Mubarak, S. (1993) Congenital muscular torticollis: Sequela of intrauterine or perinatal compartment syndrome. Journal of Pediatric Orthopaedics, 13, 141-147. 